\title{
Multi-stock portfolio optimization under prospect theory
}

\author{
Traian A. Pirvu • Klaas Schulze
}

Received: 24 August 2011 / Accepted: 23 May 2012 / Published online: 21 June 2012

(C) Springer-Verlag 2012

\begin{abstract}
We study how a behavioral agent allocates her portfolio. We consider a cumulative prospect theory investor in a single period setting with one riskless bond and multiple risky stocks, which follow a multivariate elliptical distribution. Our main result is a two-fund separation between the riskless bond and a mean-variance-portfolio, up to an exogenous benchmark portfolio. The mean-variance-portfolio, which we derive explicitly, is the same for all agents. Individual risk preferences are mirrored only in the participation in this portfolio. This dependence is illustrated by considering empirical returns. Furthermore we solve ill-posed optimization problems by imposing a regulatory risk constraint. Finally we address specific parameterizations of the value function by studying power, linear, and exponential utility.
\end{abstract}

Keywords Portfolio allocation · Prospect theory · Two-fund separation ·

Elliptical distributions

JEL Classification G81 - G11 - D03

\section{Introduction}

Markowitz [27] initiates modern portfolio theory by trading off risk and expected return of a portfolio in a one period model. Investment practice was revolutionized by his work and his message that assets should be selected in a way that takes the correlation with the other

\footnotetext{
T. A. Pirvu

Department of Mathematics \& Statistics, McMaster University, 1280 Main Street West, Hamilton, ON L8S 4K1, Canada

e-mail: tpirvu@math.mcmaster.ca

K. Schulze $(\bowtie)$

Swiss Finance Institute, Ecole Polytechnique Fédérale de Lausanne, Extranef 244,

Quartier UNIL-Dorigny, 1015 Lausanne, Switzerland

e-mail: klaas.schulze@epfl.ch
} 
securities into account. His framework provides the theoretical background for the importance of diversification, as he shows that diversification is a powerful mean of risk reduction. The Markowitz model uses a representative investor which maximizes expected utility of mean-variance type. Since his paper, the predominant optimality criterium underlying portfolio choice is expected utility maximization.

Beyond portfolio theory, expected utility theory is also accepted as the standard normative model of rational choice. It builds on the assumption that agents facing risky alternatives evaluate wealth according to final absolute outcomes, and that they are able to objectively assess probabilities. Furthermore agents are usually assumed to be uniformly risk averse. Expected utility has further been applied as a descriptive model of economic behavior under risk. However, there is substantial empirical and experimental evidence that human behavior deviates from the implications of expected utility, e.g. by paradoxa of Allais [1] or Ellsberg [15]-type or by the observation of subjective probabilities. These violation of expected utility axioms, namely the independence axiom, led to the development of several alternative paradigma of choice with higher descriptive power. One of the most prominent alternatives is Kahneman and Tversky's [22,33] prospect theory and the subsequent cumulative prospect theory (in short CPT). Roughly speaking, CPT incorporates real human decision patterns and psychology into choice behavior. Benartzi and Thaler [5] find a CPT setting with an investment horizon of one year to be plausible in explaining the famous equity premium puzzle.

This paper analyzes how such a behavioral CPT-agent optimizes her portfolio. This optimization problem has been recently solved by Bernard and Ghossoub [6] and He and Zhou [17] in a one-period setting with one riskless and one risky asset. We solve this static problem in the presence of $n$ risky choices, corresponding to a multi-stock economy. This generalization is significant, since several stocks are more realistic and allow for the essential feature of diversification.

We derive the optimal investment and, for simplicity, we state it relative to an exogenous benchmark portfolio. Our first main result is a two-fund separation of optimal excess investment between the riskless bond and a so-called mean-variance-portfolio, which is a fixed mixture of the $n$ risky stocks. All CPT-agents invest into the same mean-variance-portfolio, independent of the individual risk preferences (which only enter the individual amount invested in this mean-variance-portfolio). Our second main result is deriving this mean-variance-portfolio explicitly. The individual optimal portfolio is thus provided explicitly up to solving a one-dimensional problem. Thus we escape the curse of dimensionality and improve the computational tractability. This semi-closed form solution allows further analytical and numerical analysis of portfolio optimization, as e.g. testing model parameters empirically or considering their sensitivities as in He and Zhou [17] or establishing a connection to performance measures as in Bernard and Ghossoub [6]. It can further provide insights into modeling issues, into guiding practical portfolio investment, and into explaining financial puzzles.

Deriving the two-fund separation and the mean-variance-portfolio explicitly is appealing and maybe expected to some degree, though it is not trivial. Whereas optimal portfolios have so far been derived by maximizing expected utility, which is a concave function; the problem here is the non-concavity and non-smoothness of the prospect value criterium. The non-concavity arises from the partly non-concave 'S-shaped' utility function and the nontrivial probability distortions. Together they pose a major challenge and standard optimization techniques as convex duality and Lagrange multipliers fail. We tackle this challenge in two approaches: First, under the assumption that the optimal portfolio is finite, we derive its semi-closed form. Second, we drop this assumption. This generality comes at the price that 
the prospect value can indeed explode for large portfolios. This issue of ill-posedness is a shortcoming of prospect theory and it has sometimes been overlooked in the literature. Here prospect theory sets wrong incentives, as the trade-off between gains and losses is not present. The agent strives for an infinite portfolio. By the infinite portfolio he is exposed to infinite risk. As this excessive risk taking is not desirable, our solution is to restrict the risk of the portfolio by an additional risk constraint in terms of a fairly general risk measure. This can be justified as the internal risk assessment of CPT sets wrong incentives and is too weak in this case. In practice, the external risk measure could be enforced by a regulatory instance. The additional risk constraint restricts optimization to a compact set, and we are able to solve ill-posed problems. It turns out that agents keep the same portfolio composition and adjust only their participation when being forced to reduce risk by a binding risk constraint. We discuss in the body of the paper how this can be interpreted in favor of financial regulation.

Our CPT framework is fairly general in all its three key elements. First, we allow for general reference points or targets. We cover fixed reference points, which include the riskless return. The reference point may depend on the amounts the agents chooses to invest, which includes the expected return. It may also depend on any combination of actual asset returns, so stochastic reference points are allowed. This is important in practice as institutional investors are often evaluated relative to random benchmarks, e.g. stock indices. Second, we consider general distortion functions, as long as the prospect value is well-defined. Third, we allow for general 'S-shaped' value functions. In this CPT framework we derive the two-fund separation and the mean-variance-portfolio.

Subsequently we analyze three common choices of the value function: First, for the prominent original specification by a piecewise power value function, we derive a condition on the power utility exponents, which ensures the well-posedness of the selection problem. Further we derive the optimal portfolio in closed form. Both results are derived in similar form by $\mathrm{He}$ and Zhou [17] in the one-dimensional case. Second, for piecewise linear value functions we find a threshold on loss aversion, which ensures well-posedness. Here, we also provide the optimal portfolio explicitly, and it turns out to be the benchmark portfolio from the reference point. Third, we consider a piecewise exponential value function, which can be more adequate compared to the classic power specification, see e.g. Blondel [10], Köbberling and Wakker [23], and De Giorgi et al. [12]. Recently Rieger and Bui [31] show that this specification mimics extreme risk aversion and decision on large-scale lotteries better than the piecewise power specification. Here we derive a threshold on loss aversion ensuring well-posedness. This threshold has appealing sensitivities to the exponential utility exponents.

We assume asset returns to follow a multivariate elliptically symmetric distribution. Elliptical distribution have appealing properties and play a central role in portfolio theory. The elliptical class contains numerous common distributions, e.g. normal, $t$, exponential power, logistic, normal-variance mixtures, and symmetric generalized hyperbolic distributions. In particular the $t$ distributions, featuring fat tails and leptokurtosis, has been proved to have an excellent fit to asset returns throughout the empirical literature. We explain the elliptical distribution in detail in the body of the paper and comment on its accurate fit.

Let us briefly review the related literature without being complete. Separation results have a long history in financial economics and reach back to Tobin [32]. It is well known that two-fund separation follows in the case when expected utility is a function of portfolio's mean and variance only. Owen and Rabinovitch [28] show portfolio separation for the special cases of quadratic utilities or elliptical returns. On the side of distributions, Chamberlain [11] finally prove that the distribution of every portfolio is determined by its mean and variance if and only if the returns are elliptical. However, separation results are less known under prospect theory. Barberis and Huang [3] establish a separation result under CPT with a 
piecewise power value function and Tversky-Kahnemann-distortions for the case of normal asset returns. De Giorgi et al. [13] recently show the existence of a two-fund separation in an appealingly general reward-risk setup. This setup includes well-posed CPT problems with a piecewise positive homogeneous value function and reference points which are positive homogeneous and positive translation invariant. For clarity let us mention that the risk measure in De Giorgi et al. [13] equals the prospect loss integral in the CPT case and does not play the role of our external risk measure fixing the ill-posedness issue. Moreover, their result is rather theoretical, since it states the mere existence of a separation.

Our explicit derivation of the optimal portfolio for power utilities is inspired by Bernard and Ghossoub [6] and $\mathrm{He}$ and Zhou [17]. Both recent papers address the optimal investment of a CPT-agent into one risky stock besides deriving further findings. The main difference of the optimization problem to our paper is that we generalize to $n$ risky stocks, which is of course more realistic and allows for diversification. In their path-breaking analysis Bernard and Ghossoub [6] consider a piecewise power value function and Tversky-Kahnemann-distortions. They further impose short-selling restrictions, and borrowing-restrictions in part of their paper, whereas we generally allow for short-selling and borrowing. Depending on the returns both features are needed to compose the optimal multi-dimensional portfolio. He and Zhou [17] consider general 'S-shaped' value functions for a fixed reference point and appealingly find conditions under which the optimal investment problem is well-posed. In the case the problem is ill-posed, we are still able to solve it by imposing the external risk constraint. Moreover, He and Zhou [17] derive explicitly the one-dimensional optimal portfolio for piecewise power and piecewise linear utilities. De Giorgi et al. [12] also consider the illposedness issue and show that it is avoided for exponential utilities and normal asset returns.

In order to illustrate our analysis we compute the optimal portfolio for empirical returns of five major US stocks. We build on the estimation of Hu and Kercheval [18] and observe that the total amount agents with a piecewise power value function invest into the five stocks is generally very high. Further we find that this amount is increasing in the power utility exponent on gains and decreasing in the power exponent on losses, which is consistent with intuition. Moreover, we find that this amount is much higher for normal returns compared to returns following a $t$ distribution. This indicates that piecewise power agents can be very sensitive to the tail behavior of the returns. Finally, we consider the total amount invested by piecewise exponential CPT-agents and it turns out to be generally lower than for piecewise power agents. This could be explained by the high risk aversion to large-scale lotteries of piecewise exponential agents. Again we find that the amount of total investment has appealing sensitivities to exponential utility exponents.

This paper is organized as follows: Section 2 defines the behavioral setting with a CPTagent and elliptical returns. Section 3 derives the optimal portfolio in the unconstrained and constrained case. In Section 4 we consider common choices of the value function, and Section 5 presents the numerical illustration. All proofs are delegated to the appendix.

\section{The setting}

\subsection{Prospect theory}

In this section we formalize the one-period portfolio choice problem and the behavioral agent under CPT (in short CPT-agent). Whereas Tversky and Kahneman [33] consider discrete probability distributions, we adopt the notation of Bernard and Ghossoub [6] who, similar as He and Zhou [17], generalize to continuous distributions. 
The financial market of the portfolio choice problem consists of one risk-free asset, with return $r$ over the period, and $n$ risky assets with stochastic returns $x=\left(x_{1}, \ldots, x_{n}\right)^{T}$. The amount $\pi_{i}$ is invested in the risky asset $x_{i}$ and the remaining wealth $W_{0}-\sum_{i=1}^{n} \pi_{i}$ is invested in the risk-free asset. The individual's wealth at the end of the period is given by

$$
W=\left(W_{0}-\sum_{i=1}^{n} \pi_{i}\right)(1+r)+\sum_{i=1}^{n} \pi_{i}\left(1+x_{i}\right)=W_{0}(1+r)+\sum_{i=1}^{n} \pi_{i} \bar{x}_{i},
$$

where the $\bar{x}=\left(\bar{x}_{1}, \ldots, \bar{x}_{n}\right)^{T}$ denotes the vector of excess returns on the risky assets over the risk-free asset, i.e.

$$
\bar{x}:=x-r \mathbf{1}
$$

Kahneman and Tversky $[22,33]$ conduct several simple experiments which lead to the following conclusions about human behavior, which constitute the three key elements of CPT:

1. Agents evaluate outcomes in comparison to a certain benchmark, target or anchor, rather than on absolute final wealth. This behavior is modeled by a reference point, which divides outcomes into gains and losses. We formalize this reference point of wealth $W^{\text {ref }}$ by defining

$$
W^{\mathrm{ref}}:=a \cdot \bar{x}+b \cdot \pi+p,
$$

for some vectors $a, b \in \mathbb{R}^{n}$, and a scalar $p$, and where

$$
a \cdot b:=\sum_{i=1}^{n} a_{i} b_{i}
$$

denotes the scalar product. We allow for linear combinations of actual investment $\pi$, stochastic returns $\bar{x}$, and fixed levels $p$. We briefly list some simple examples:

(a) Setting $a, b=\mathbf{0}$ gives a fixed reference point. An important special case of a fixed reference points is $p=W_{0}(1+r)$, the risk-free return.

(b) Setting $a=\mathbf{0}, b=E \bar{x}, p=W_{0}(1+r)$, where $E \bar{x}$ denotes the mean vector of $\bar{x}$, gives the expected wealth $W^{\text {ref }}=E W$.

(c) Setting $a=\frac{100}{n} \cdot \mathbf{1}, b=\mathbf{0}, p=\frac{100}{n}(1+r)$ gives an equally weighting index of stochastic assets values $W^{\text {ref }}=\frac{100}{n} \sum_{i=1}^{n}\left(1+x_{i}\right)$.

Instead of focusing on total investment amounts $\pi$, we concentrate on investment in excess of the benchmark portfolio $a$ in the reference point and set

$$
\zeta:=\pi-a .
$$

This simplifies the presentation and we parametrize portfolios in terms of excess investment $\zeta$. Notice that total investment can easily be recovered by $\pi=\zeta+a$ in the less common case of $a$ not equaling 0 , see also Remark 1 . Further we set $y$, the excess return over risk-free rate and benchmark return in the reference point, and the number $c$ by

$$
y:=\bar{x}-b, \quad c:=p+a \cdot b-W_{0}(1+r) .
$$

These definition give that the actual random wealth at the end of the period is $W=$ $W^{\text {ref }}+\zeta \cdot y-c$. Thus the deviation from the reference point is given by

$$
D(\zeta)=W-W^{\mathrm{ref}}=\zeta \cdot y-c .
$$


The one-dimensional cumulative density function of the crucial random variable $D(\zeta)$ is denoted by $F$ and is addressed in detail in the Subsect. 2.2.

2. Instead of being uniformly risk-averse over all outcomes, agents behave differently on gains and losses and are furthermore loss-averse, i.e. significantly more sensitive to losses than to gains. This is modeled by replacing the concave utility function by a 'S-shaped' value function, which is concave for gains, but convex for losses, and furthermore steeper for losses than for gains. This value function is defined by

$$
u(x):= \begin{cases}u^{+}(x) & \text { for } x \geq 0 \\ u^{-}(-x) & \text { for } x \leq 0\end{cases}
$$

where $u^{+}: \overline{\mathbb{R}}^{+} \rightarrow \overline{\mathbb{R}}^{+}$and $u^{-}: \overline{\mathbb{R}}^{+} \rightarrow \overline{\mathbb{R}}^{+}$are differentiable, increasing, and convex functions.

3. Agents have preferences, which transform objective probabilities; they overweight small probabilities and underweight large probabilities. This is modeled by probability distortion functions $T^{+}:[0,1] \rightarrow[0,1]$ for gains and $T^{-}:[0,1] \rightarrow[0,1]$ for losses, which increase small probabilities and shrink large probabilities. Tversky and Kahneman [33] suggest the following functional form for the distortions

$$
T^{+}(p):=\frac{p^{\gamma}}{\left[p^{\gamma}+(1-p)^{\gamma}\right]^{1 / \gamma}}, \quad T^{-}(p):=\frac{p^{\delta}}{\left[p^{\delta}+(1-p)^{\delta}\right]^{1 / \delta}} .
$$

Ingersoll [21] shows that $\gamma, \delta>0.28$ ensures that $T^{+}, T^{-}$are increasing and Tversky and Kahneman [33] suggest the estimates $\gamma=0.61$ and $\delta=0.69$. Prelec [30] suggests the distortions

$$
T^{+}(p):=e^{-\delta_{1}(-\log p)^{\gamma}}, \quad T^{-}(p):=e^{-\delta_{2}(-\log p)^{\gamma}} .
$$

Overall the prospect value of the portfolio is thus given by the difference of the prospect value of gains and losses, weighted by the parameter of loss aversion $\lambda>0$

$$
\begin{aligned}
V(D(\zeta)) & :=V^{+}(D(\zeta))-\lambda V^{-}(D(\zeta)), \quad \text { where } \\
V^{+}(D(\zeta)) & :=\int_{0}^{\infty} u^{+}(x) d\left[-T^{+}(1-F(x))\right], \\
V^{-}(D(\zeta)) & :=\int_{-\infty}^{0} u^{-}(-x) d\left[T^{-}(F(x))\right]
\end{aligned}
$$

Finally, we require that the prospect value $V(D(\zeta))$ is well defined.

Assumption 1 The prospect values of gains $V^{+}(D(\zeta))$ and losses $V^{-}(D(\zeta))$ are finite for all $\zeta \in R^{n}$.

The following proposition, which is a slight generalization of a condition in He and Zhou [17] states, which combinations of distortion functions and return distributions ensure that the prospect value is well-defined.

Proposition 1 If asset returns y have a normal, lognormal or $t$ distribution and for sufficiently small $x>0$ there exists some $\eta$ with $0<\eta<1$ and 


$$
\begin{aligned}
T_{x}^{+}(x)=O\left(x^{-\eta}\right), & T_{x}^{-}(x)=O\left(x^{-\eta}\right), \\
T_{x}^{+}(1-x)=O\left(x^{-\eta}\right), & T_{x}^{-}(1-x)=O\left(x^{-\eta}\right) .
\end{aligned}
$$

then Assumption 1 is satisfied.

Note that both distortion functions (2) and (3) satisfy (5).

\subsection{Distribution of returns}

Assumption 2 The random vector $y=\left(y_{1}, \ldots, y_{n}\right)^{T}$ of excess returns on the risky assets, defined in (1), has an elliptically symmetric distribution.

We assume that the vector of means $E(y)=\mu=\left(\mu_{1}, \ldots, \mu_{n}\right)^{T}$ and the $n \times n$ covariance matrix $\operatorname{Cov}(y)=\Sigma=\left(\sigma_{i j}\right)_{i, j}$ exist and that $\Sigma$ is positive definite. Further we assume that the density $f$ exists. Then it takes the form

$$
f(x)=|\Sigma|^{-\frac{1}{2}} g\left[(x-\mu)^{T} \Sigma^{-1}(x-\mu)\right],
$$

where $x \in \mathbb{R}^{n}$ and $g: \mathbb{R}^{+} \rightarrow \mathbb{R}^{+}$is called density generator or shape of $y$, and we write

$$
y \sim E C_{n}(\mu, \Sigma ; g),
$$

where $(\mu, \Sigma)$ is called parametric part and $g$ is called the non-parametric part of the elliptical distribution. The characteristic function $\psi$ of $y$ has the form

$$
\psi_{y}(t)=E \exp \left(i t^{T} y\right)=\exp \left(i t^{T} \mu\right) \phi\left(t^{T} \Sigma t\right),
$$

for some scalar function $\phi$, called the characteristic generator. For background on the elliptically symmetric distribution, which is also called elliptically countered, see Fang, Kotz, and $\mathrm{Ng}$ [16] and Bingham and Kiesel [7].

Elliptical distributions are appealing for portfolio analysis, since linear combinations of elliptical random vectors are elliptical itself. In particular, we use that the deviation from the reference level $D(\zeta)$ is univariate elliptically distributed, i.e.

$$
D(\zeta)=\zeta \cdot y-c \sim E C_{1}(\bar{\mu}, \bar{\sigma} ; g)
$$

where the density generator $g$ remains unchanged and the mean and variance equal

$$
\bar{\mu}:=E D(\zeta)=\zeta \cdot \mu-c, \quad \bar{\sigma}^{2}:=E(D(\zeta)-\bar{\mu})^{2}=\zeta^{T} \Sigma \zeta=\sum_{i=1}^{n} \sum_{j=1}^{n} \zeta_{i} \zeta_{j} \sigma_{i j},
$$

which holds throughout the paper. A second appealing fact for portfolio analysis is that the non-parametric part $g$ escapes the curse of dimensionality, see Bingham and Kiesel [7]: dimension $n$ is the number of stocks and may be very large. Whereas this dictates the dimensions of the parametric part $(\mu, \Sigma)$ in order to retain the Markowitzian interpretations, the crucial non-parametric part $g$ is a one-dimensional function independent of $n$. This allows simulation studies of elliptical models to be computational tractable.

Hence elliptical distributions play a central role in portfolio analysis. They are chosen to model the stock returns e.g. by Chamberlain [11], Owen and Rabinovitch [28], and Bingham et al. [8]. Chamberlain [11] proves that expected utility of a portfolio is determined solely by its mean and variance if and only if asset returns are elliptical. We conjecture that this result extends to CPT. Our proofs rely heavily on the fact that, for a fixed generator, the distribution of the deviation is determined by its mean and variance only, given in Eq. (7). We doubt that 
this fact and the derivation of our results extend beyond elliptical distributions, which closes our results to some degree to the elliptical class.

Fortunately, the class of elliptical distributions contains many common distributions of asset returns. Note that the following distributions are elliptical: the multivariate normal distribution, the multivariate Cauchy distribution, the multivariate $t$ distribution, the multivariate exponential power family (and thus the Laplace distribution), the multivariate logistic family, normal-variance mixture distributions, symmetric stable distributions, the symmetric variance-gamma distribution, the symmetric normal inverse Gaussian distribution, and more generally the symmetric generalized hyperbolic distribution. Let us consider the following special cases of the elliptical distribution more detailed:

1. Normal distribution. Setting the density generator to

$$
g(u)=(2 \pi)^{-\frac{n}{2}} \exp \left(-\frac{1}{2} u\right)
$$

yields the multivariate normal distribution with mean vector $\mu$ and covariance matrix $\Sigma$, so

$$
y \sim N_{n}(\mu, \Sigma)
$$

Hence the deviation $D(\zeta)$ is univariate normal, i.e. $D_{N}(\zeta) \sim N_{1}(\bar{\mu}, \bar{\sigma})$.

2. Student's $t$ distribution. Setting the density generator to

$$
g(u)=(\pi v)^{-\frac{n}{2}} \frac{\Gamma(N)}{\Gamma(N-n / 2)}\left(1+\frac{u}{v}\right)^{-N},
$$

where $N>\frac{n}{2}$ and $v>0$ yields the multivariate $t$ distribution with mean vector $\mu$, covariance matrix $\Sigma$, and $v$ degrees of freedom, so

$$
y \sim t_{n}(\mu, \Sigma, v) .
$$

Thus it can be decomposed by $y=\frac{A Z}{\sqrt{v^{-1} C}}+\mu$, where $Z$ is multivariate standard normally distributed and independent of $C$, which is $\chi^{2}$-distributed with $v$ degrees of freedom. Further the matrix $A$ is given by the Cholesky decomposition $A A^{T}=\Sigma$. Notice that $\zeta A Z$ is univariate normal, i.e. $\zeta A Z \sim N(0, \bar{\sigma})$, by

$$
\operatorname{Var}(\zeta A Z)=\zeta^{T} \operatorname{Var}(A Z) \zeta=\zeta^{T} A I_{n} A^{T} \zeta=\zeta^{T} \Sigma \zeta=\bar{\sigma}^{2} .
$$

Thus the ratio $\frac{\zeta A Z}{\sqrt{v^{-1} C}}$ is univariate $t$ distributed. In turn the deviation $D(\zeta)$ is univariate $t$ distributed with mean $\bar{\mu}$ and variance $\bar{\sigma}^{2}$, i.e.

$$
D(\zeta)=\zeta \cdot y=\frac{\zeta A Z}{\sqrt{v^{-1} C}}+\zeta \cdot \mu \sim t_{1}(\bar{\mu}, \bar{\sigma}, v)
$$

Note that for $v>2$ it holds $D(\zeta) \in L_{2}$.

3. Logistic distribution. Setting the density generator to

$$
g(u)=(2 \pi)^{-\frac{n}{2}}\left[\sum_{j=1}^{\infty}(-1)^{j-1} j^{1-n / 2}\right]^{-1} \frac{e^{-u}}{\left(1+e^{-u}\right)^{2}}
$$

yields the multivariate logistic distribution with mean vector $\mu$ and covariance matrix $\Sigma$, so

$$
y \sim M L_{n}(\mu, \Sigma)
$$

Hence the deviation $D(\zeta)$ is univariate logistic, i.e. $D_{N}(\zeta) \sim M L_{1}(\bar{\mu}, \bar{\sigma})$. 
4. Normal-variance mixtures. The random vector $y$ is called a multivariate normal-variance mixture, if

$$
y \sim \mu+\sqrt{U} Z,
$$

where $\mu$ is the mean vector, $Z$ is multivariate normal with zero-mean and covariance matrix $\Sigma^{\prime}$, i.e. $Z \sim N_{n}\left(0, \Sigma^{\prime}\right)$, and $U$ is a random variable on $(0, \infty)$ with cdf $F_{U}$. It follows that $E(y)=\mu$ and $\operatorname{Cov}(y)=E U \Sigma^{\prime}=: \Sigma$. It follows further that, conditioned on the event $U=u$, the distribution of $y$ is normal

$$
y \mid(U=u) \sim N_{n}(\mu, u \Sigma) .
$$

The so called mixing variable $U$ can be interpreted as as shock, which changes the variance of an underlying normal distribution. These mixtures have been studied e.g. in Barndoff-Nielsen et al. [4]. Their characteristic function $\psi$ takes the form

$$
\psi_{y}(t)=\exp \left(i t^{T} \mu\right) \phi_{M}\left(\frac{1}{2} t^{T} \Sigma t\right),
$$

where $\phi_{M}$ is the one-sided Laplace-transform of $U$. Comparing with (6), it follows that all normal-variance mixtures are elliptical with characteristic generator $\phi(s)=$ $\phi_{M}(s / 2)$. Conversely, any elliptical distribution whose characteristic generator is completely monotone is a normal-variance mixture.

The class of normal-variance mixtures contains several common distributions. A constant mixing variable $U$ yields the multivariate normal distribution for $y$. Considering a discrete distribution for $U$ yields a weighted average of normal distribution for $y$. In particular Hull and White [19] use the Bernoulli distribution for $U$. Setting $F_{U}$ to the inverse Gaussian distribution gives rise to the multivariate $t$ distribution. Finally, mixing by the gamma distribution gives the symmetric variance gamma distribution, and more generally, mixing by the generalized inverse Gaussian distribution yields the symmetric generalized hyperbolic distribution.

Finally, many elliptical distributions provide an accurate fit to real-world data of stock returns. Let us refer exemplary to the following empirical studies: Eberlein and Keller [14] introduce the hyperbolic distribution for daily asset returns and find a good fit, when the distribution is nearly symmetric, and thus elliptical. Subsequently the authors concentrate on the symmetric (elliptical) case for a Levy motion model. Madan and Seneta [26] introduce the multivariate symmetric variance gamma distribution and fit it successfully to stock returns. Student's $t$ distribution has been proved throughout the literature to fit asset returns very well, e.g. by Praetz [29], Blattberg and Gonedes [9], Aparicio and Estrada [2], and by $\mathrm{Hu}$ and Kercheval [18] in the multivariate case. In particular Hurst and Platen [20] study the empirical fit of normal mean-variance mixtures, as they consider: the logstable model by Mandelbrot and Fama; the Clark model; the symmetric generalized hyperbolic model by various authors; the $t$ model by Blattberg and Gonedes [9] and Praetz [29]; the lognormalinverse Gaussian model by Barndorff-Nielsen; the hyperbolic model by Kuechler, and by Eberlein and Keller [14]; and the variance gamma model by Madan and Seneta [26]. Hurst and Platen [20] perform their empirical analysis with the data of daily returns of the indexes of five major market over 15 years. The goodness of fit of all mentioned models is compared by ratio of the likelihood of the classical lognormal model over the likelihood of the tested model. The $t$ model turns out to achieve the best fit and outperforms all other models. This result is robust over all five markets and over the exclusion of extreme events. Intuitively, the $t$ law has the largest probability for the observed return data and adds the most information 
with the minimum number of parameters compared to the normal distribution. Finally, Levy and Duchin [25] compare the fit of 11 distributions, including various non-elliptical distributions. The logistic distribution (which is elliptical) outperforms surprisingly strongly all other distributions for varying horizons up to a year. Levy and Duchin [25] state "For relatively short horizons, which probably cover the most relevant horizons for most investors (one day to one year), the logistic distribution [...] clearly dominates. This is true for individual stocks as well as portfolios."

\section{Optimal portfolios}

In this section we solve the optimization problem of maximizing the prospect value of (4):

$$
\sup _{\zeta \in \mathbb{R}^{n}} V(D(\zeta))
$$

We derive our main result of a two-fund separation, first under the assumption that the optimal portfolio is finite, and then without this assumption by restricting portfolios by a risk constraint. In our setting we allow for borrowing, i.e. $\sum_{i=1}^{n} \zeta_{i}>W_{0}$, and unlike in Bernard and Ghossoub [6] short-selling, i.e. $\zeta_{i}<0$, is also allowed.

\subsection{Unconstrained case}

In this section we assume that the optimal portfolio $\zeta^{*}$ exists, which implies that it is finite.

Assumption 3 There exists $\zeta^{*} \in \mathbb{R}^{n}$ with

$$
\zeta^{*}=\arg \max _{\zeta \in \mathbb{R}^{n}} V(D(\zeta)) .
$$

This optimal portfolio composition $\zeta^{*}$ is derived in the following theorem. It shows that is optimal for agents to invest in the risk-free asset and a portfolio of risky assets, called meanvariance-portfolio. Note that the composition of the mean-variance-portfolio is the same for all CPT-agents. It is independent of their individual risk preferences. It does depend only on the means and correlation of the $n$ risky assets. The mean-variance-portfolio composition $\zeta_{M}$ is defined by

$$
\zeta_{M}:=\Sigma^{-1} \mu .
$$

The mean-variance-portfolio gives a deviation of

$$
D\left(\zeta_{M}\right)=\zeta_{M} \cdot y-c=\Sigma^{-1} \mu \cdot y-c .
$$

Note that the mean of the mean-variance-portfolio differs from its variance only by the number $c$ :

$$
\begin{aligned}
E D\left(\zeta_{M}\right)+c & =E\left(\zeta_{M} \cdot y-c\right)+c=\zeta_{M} \cdot \mu=\zeta_{M}^{T} I_{n} \mu \\
& =\zeta_{M}^{T} \Sigma \Sigma^{-1} \mu=\zeta_{M}^{T} \Sigma \zeta_{M}=\operatorname{Var}\left(D\left(\zeta_{M}\right)\right)=: \sigma_{M}^{2}
\end{aligned}
$$

Let us define the optimal participation level in the mean-variance-portfolio

$$
k^{*}:=\arg \max _{k \in \mathbb{R}} V\left(D\left(k \zeta_{M}\right)\right) .
$$


Theorem 4 Under Assumption 3 the optimal portfolio of the CPT-agent is given by

$$
\zeta^{*}=k^{*} \zeta_{M}=k^{*} \Sigma^{-1} \mu \text {. }
$$

Hence the optimal total investment is given by $\pi^{*}=k^{*} \zeta_{M}+a$.

Remark 1 For many reference points, e.g. risk-free return and expected value, the benchmark portfolio $a$ equals zero. In this case excess investment $\zeta$ and total investment $\pi$ coincide, and the two-fund separation also holds for total investment by $\pi^{*}=k^{*} \zeta_{M}$.

This theorem has two implications: It provides the mean-variance-portfolio explicitly and it establishes the two-fund separation for well-posed CPT selection problems up to the exogenous benchmark portfolio $a$. This gives a semi-closed form of the optimal total investment $\pi^{*}$ : In particular theorem provides that the optimal total investment is necessarily of the form

$$
\pi=k \zeta_{M}+a
$$

for some $k \in \mathbb{R}$. This portfolio gives a deviation of

$$
W-W^{r e f}=(\pi-a) \cdot y-c=k \zeta_{M} \cdot y-c=D\left(k \zeta_{M}\right) .
$$

Hence it only remains to solve for $k^{*}$ as defined in (12). Consequently the $n$-dimensional portfolio optimization problem is reduced to a one-dimensional optimization and we escape the curse of dimensionality. This is appealing for traders who optimize high-dimensional portfolios. The semi-closed-form solution allows further analytical and numerical analysis of portfolio optimization, as e.g. testing model parameters empirically or considering their sensitivities as in He and Zhou [17] or establishing a connection to performance measures as in Bernard and Ghossoub [6]. They can further provide insights into modeling issues, into guiding practical portfolio investment, and into explaining financial puzzles.

\subsection{Constrained case}

In this section we are not imposing Assumption 3. The price we pay for this generality is that it is no longer ensured the maximization problem is well-posed. In an ill-posed problem the maximal prospect value is not gained by a finite portfolio. It sets wrong incentives as the agent strives for an infinite portfolio, and thus infinite prospect value, since the trade-off between gains and losses is perverted. This ill-posedness issue is a shortcoming of prospect theory, whereas it is less severe in the classical portfolio choice models of expected utility. He and Zhou [17] analyze this issue and derive conditions on the modeling parameters to ensure well-posedness. They are able to state the cut-off between well-posed and ill-posed models in terms of their definition of loss aversion.

Our approach is different, as we aim to solve the problem regardless of its wellposedness. In an ill-posed problem, the agent has wrong incentives to blow up his position in the risky assets. The optimal infinite portfolio would be exposed to infinite risk. Since this is not favorable, our approach is to restrict the risk of a portfolio by a risk measure constraint. This avoids harmful excessive risk taking. In practice this corresponds to a supervising agency regulating the agent by imposing a certain level of maximal risk. Prospect theory already accounts for risk by the disutility from losses. However, for the ill-posed models this internal risk measure is too weak as it provides wrong incentives. This can be resolved by imposing an external risk measure, which bounds risk effectively. It is obvious and known in the literature that an arbitrary binding constraint ensures well-posedness. It is also quite obvious to choose a risk measure here, nonetheless we believe that considering risk 
measures in this context is probably new in the literature. This choice is economically very appealing, as just argued. It is further mathematically fortunate, as we are fairly general with respect to the risk measure, and the natural derivatives of prospect value and risk measures with respect to the mean allow us to solve the ill-posed problems, and to provide semi-closed forms of optimal portfolios.

In this section we consider the constrained problem

$$
\sup _{\zeta \in K} V(D(\zeta))
$$

where $K$ is the set of admissible portfolios. A portfolio is admissible if its risk, measured by a risk measure $\rho$, is at most $M$, i.e.

$$
K:=\left\{\zeta \in \mathbb{R}^{n}: \rho(D(\zeta)) \leq M\right\} .
$$

For the risk measure $\rho$ we allow any $C^{1}$-function $\rho=\rho(D(\zeta))=\rho(\bar{\mu}(\zeta), \bar{\sigma}(\zeta))$, which depends only on the portfolio mean $\bar{\mu}$ and standard deviation $\bar{\sigma}$, has derivatives $\rho_{\bar{\mu}}<0$ and $\rho_{\bar{\sigma}}>0$, and

$$
\lim _{|\zeta| \rightarrow \infty} \rho(\bar{\mu}(\zeta), \bar{\sigma}(\zeta))=\infty
$$

This last condition implies that the constraint set $K$ is compact. The two conditions on the partial derivatives are natural for a risk measure: A risk measure should be decreasing in the mean $\bar{\mu}$ and increasing in the standard deviation $\bar{\sigma}$. Note that the above conditions hold true e.g. for Value-at-Risk and Average-Value-at-Risk, given that the confidence level $a$ is small enough. This is very appealing as general law-invariant convex risk measures can be constructed by using Average-Value-at-Risk as building blocks. The first proof of this representation is given by Kusuoka [24] in the coherent case. The two mentioned risk measures can be written as

$$
\begin{gathered}
\operatorname{VaR}_{a}(\bar{\mu}, \bar{\sigma})=-\bar{\mu}-\bar{\sigma} \Theta^{-1}(a), \\
A \operatorname{VaR}_{a}(\bar{\mu}, \bar{\sigma})=-\bar{\mu}-\frac{\bar{\sigma}}{a} \int_{0}^{a} \Theta^{-1}(s) d s,
\end{gathered}
$$

where $\Theta^{-1}$ denotes the inverse of the standardized cdf $\Theta(x):=F(\bar{\sigma} x+\bar{\mu})$ of the deviation. Indeed, since $\bar{\sigma} \geq \sqrt{q}|\zeta|$ and $\bar{\mu} \leq|\mu||\zeta|$ it is enough to choose $a$ such that $-F^{-1}(a) \geq \frac{|\mu|}{q}$ for (15) to hold true for $V a R$ and $A V a R$.

In order to state the optimal portfolio we define the set of admissible participation levels in the mean-variance-portfolio

$$
L:=\left\{k \in \mathbb{R}: \rho\left(D\left(k \zeta_{M}\right)\right) \leq M\right\},
$$

and the optimal admissible participation level

$$
\tilde{k}:=\arg \max _{k \in L} V\left(D\left(k \zeta_{M}\right)\right) .
$$

Theorem 5 The optimal portfolio of the CPT-agent, who is constrained by the set $K$

$$
\zeta_{K}^{*}:=\arg \max _{\zeta \in K} V(D(\zeta))
$$

it is given by

$$
\zeta_{K}^{*}=\tilde{k} \zeta_{M}
$$


This implies the optimal total investment of $\pi_{K}^{*}=\tilde{k} \zeta_{M}+a$.

This Theorem implies the two-fund separation and the semi-closed form of the optimal portfolio by constraining risk externally.

Remark 2 Let us compare the constrained and the unconstrained case under the assumption that 3 holds true. In case of $\rho\left(D\left(\zeta^{*}\right)\right) \leq M$ the globally optimal portfolio $\zeta^{*}$ is admissible and the risk constraint is not binding. Hence it holds $\zeta_{K}^{*}=\zeta^{*}$ and $\tilde{k}=k^{*}$. More interesting is the case of $\rho\left(D\left(\zeta^{*}\right)\right)>M$. The global optimum is not admissible as its exposure to risk in terms of $\rho$ is too high. The agent cannot choose $\zeta_{K}^{*}=\zeta^{*}$ and is forced to reallocate. One might expect that she now overweights stocks with low $\rho$-risk-exposure and high prospect value. She might adapt to the specific form of the risk measure. Different risk measures rank portfolios differently. For example, $A V a R$ always favors diversification, whereas $\operatorname{VaR}$ may penalize diversification. However, the last theorem shows that she still keeps the fixed composition of the mean-variance-portfolio and she chooses the binding participation $\tilde{k}$ with $\rho\left(D\left(\tilde{k} \zeta_{M}\right)\right)=M$. She chooses not to adjust her portfolio composition to the specific form of the external risk constraint. The reason for this is that the mean-variance-portfolio has the optimal mean-variance-trade-off in terms of the prospect value modulo scaling of the portfolio. Any other portfolio with a higher prospect value modulo scaling is penalized by the risk measure $\rho$, which has natural sensitivities with respect to mean and variance. The absence of adjustment due to the external risk constraint can be interpreted in favor of financial regulation: The external constraint achieves its purpose of bounding risk without providing unwanted incentives to adjust the originally optimal portfolio composition.

\section{Common choices of value functions}

In this section we consider special cases of the CPT framework, as we specify the ' $\mathrm{S}$ '-shaped value function to be of the several types, which are central choices in the literature of prospect theory. While studying these special cases we set for simplicity $c=0$, meaning that the deterministic deviation at zero investment equals zero. This implies

$$
V(D(\mathbf{0}))=V(0-0)=0 .
$$

\subsection{Piecewise power value function}

We study the case of power utilities in detail, as it is the original choice in Tversky and Kahneman [33] and the most prominent specification of the value function. We define

$$
\begin{aligned}
& u^{+}(x):=x^{\alpha}, \text { where } 0<\alpha<1 \text { and } x \geq 0, \\
& u^{-}(x):=x^{\beta}, \text { where } 0<\beta<1 \text { and } x>0, \\
& u^{+}(\infty)=u^{-}(\infty)=\infty
\end{aligned}
$$

In contrast to Tversky and Kahneman [33] we allow for $\alpha>\beta$. We restate a condition of Barberis and Huang [3] on parameters $\alpha$ and $\beta$, which ensures that the prospect value is well defined.

Proposition 2 If distortion functions are of type (2) and it holds

$$
\alpha<2 \min (\delta, \gamma) \text { and } \beta<2 \min (\delta, \gamma) \text {, }
$$

then Assumption 1 is satisfied. 
In the remainder of this subsection we solve for the optimal portfolio of a CPT investor with a piecewise power value function. Depending on her power utility exponents $\alpha$ and $\beta$ her optimal portfolio is finite or not. The next proposition provides that parameters with $\alpha<\beta$ imply a finite optimal portfolio, and in consequence the two-fund separation by Theorem 4 .

Proposition 3 If $\alpha<\beta$, then it holds

$$
\lim \sup _{|\zeta| \rightarrow \infty} V(D(\zeta))=-\infty
$$

which implies Assumption 3.

In this case of $\alpha<\beta$, we can state the optimal participation level in the mean-variance-portfolio $k^{*}$ explicitly. To do so we define the cdf of the standardized deviation $D(\zeta)$ by

$$
\Theta(x):=F(\bar{\sigma} x+\bar{\mu})
$$

with $\bar{\mu}$ and $\bar{\sigma}$ defined in (8). Further we define the distorted density functions $f^{+}$and $f^{-}$of the $\operatorname{cdf} \Theta$ by the following two derivatives

$$
f^{+}(x):=-\left(T^{+}(1-\Theta(x))\right)_{x}, \quad f^{-}(x):=\left(T^{-}(\Theta(x))\right)_{x},
$$

which are independent of $\bar{\mu}$ and $\bar{\sigma}$.

Proposition 4 For $\alpha<\beta$ the optimal portfolio of the CPT-agent is given by

$$
\zeta^{*}=k^{*} \zeta_{M}= \begin{cases}k_{1} \zeta_{M}, & \text { if } V\left(D\left(k_{1} \zeta_{M}\right)\right) \geq V\left(D\left(k_{2} \zeta_{M}\right)\right), \\ k_{2} \zeta_{M}, & \text { if } V\left(D\left(k_{1} \zeta_{M}\right)\right)<V\left(D\left(k_{2} \zeta_{M}\right)\right),\end{cases}
$$

where

$$
k_{1}=\left(\frac{\alpha}{\beta}\right)^{\frac{1}{\beta-\alpha}}\left(\frac{\int_{0}^{\infty} x^{\alpha} f^{+}\left(\frac{x}{\sigma_{M}}-\sigma_{M}\right) \mathrm{d} x}{\lambda \int_{-\infty}^{0}(-x)^{\beta} f^{-}\left(\frac{x}{\sigma_{M}}-\sigma_{M}\right) \mathrm{d} x}\right)^{\frac{1}{\beta-\alpha}}<\infty,
$$

and

$$
k_{2}=-\left(\frac{\alpha}{\beta}\right)^{\frac{1}{\beta-\alpha}}\left(\frac{\int_{0}^{\infty} x^{\alpha} f^{+}\left(\frac{-x}{\sigma_{M}}-\sigma_{M}\right) \mathrm{d} x}{\lambda \int_{-\infty}^{0}(-x)^{\beta} f^{-}\left(\frac{-x}{\sigma_{M}}-\sigma_{M}\right) \mathrm{d} x}\right)^{\frac{1}{\beta-\alpha}}>-\infty .
$$

Thus optimal total investment is given by $\pi^{*}=k^{*} \zeta_{M}+a$.

For $\alpha=\beta$ Assumption 3 may be violated depending on loss aversion $\lambda$; for $\alpha>\beta$ Assumption 3 is violated. This is obvious by our proofs and worked out more detailed in He and Zhou [17]. However, without Assumption 3 a finite optimal portfolio can be found by restricting risk externally and Theorem 5 .

\subsection{Linear utilities}

We consider piecewise linear value functions. He and Zhou [17] analyze this specification in detail. Furthermore Benartzi and Thaler [5] use linear utilities when addressing the equity premium puzzle. We define

1. $u^{+}(x):=x$, where $x \geq 0$,

2. $u^{-}(x):=x$, for $x>0$,

3. $u^{+}(\infty)=u^{-}(\infty)=\infty$. 
Linear utilities are a special case of power utilities with parameters $\alpha=\beta=1$. Thus Proposition 2 gives that

$$
\frac{1}{2}<\min (\delta, \gamma)
$$

yields Assumption 1. Note that Proposition 3 and 4 do not apply for linear utilities. However, we find a threshold for loss aversion $\lambda$ that ensures the two-fund separation for CPT-agents with piecewise linear value functions.

Proposition 5 If it holds

$$
\lambda>\frac{V^{+}\left(D\left(\zeta_{d}\right)\right)}{V^{-}\left(D\left(\zeta_{d}\right)\right)},
$$

where the number $d$ is defined in (31) and $\zeta_{d}$ is a portfolio for which the deviation has mean $\bar{\mu}\left(\zeta_{d}\right)=d$ and standard deviation $\bar{\sigma}\left(\zeta_{d}\right)=1$, then Assumption 3 is satisfied.

In the other direction, we find that Assumption 3 implies a threshold for loss aversion $\lambda$. Furthermore, under Assumption 3 we solve for the optimal portfolio explicitly, and find that it is optimal for a linear CPT-agent not to deviate from the benchmark portfolio $a$.

Proposition 6 If Assumption 3 is satisfied, then it holds

$$
\lambda \geq \max \left(\frac{V^{+}\left(D\left(\zeta_{M}\right)\right)}{V^{-}\left(D\left(\zeta_{M}\right)\right)}, \frac{V^{+}\left(D\left(-\zeta_{M}\right)\right)}{V^{-}\left(D\left(-\zeta_{M}\right)\right)}\right),
$$

and the optimal portfolio of the CPT-agent is given by

$$
\zeta^{*}=\mathbf{0}
$$

Hence optimal total investment is given by $\pi^{*}=a$.

\subsection{Exponential utilities}

Recently specifying the value function as piecewise CARA utility is raising interest, for example in Köbberling and Wakker [23] and De Giorgi et al. [12]. Rieger and Bui [31] use this specification in order to better explain very risk averse behavior and decisions on large-scales lotteries. We define

1. $u^{+}(x):=\frac{1}{v}\left(1-e^{-v x}\right)$, where $0<v$ and $x \geq 0$,

2. $u^{-}(x):=\frac{1}{\xi}\left(1-e^{-\xi x}\right)$, where $0<\xi$ and $x>0$,

By the inequalities

$$
\frac{1}{v}\left(1-e^{-v x}\right) \leq \frac{1}{v} \text { and } \frac{1}{\xi}\left(1-e^{-\xi x}\right) \leq \frac{1}{\xi} \text { for } x \geq 0
$$

Assumption 1 is satisfied for general distortions and asset returns. The final proposition provides a threshold for loss aversion, which establishes the two-fund separation for CPT-agents with piecewise exponential value functions.

Proposition 7 If it holds

$$
\lambda>\frac{\xi}{v} \frac{T^{+}(1-\Theta(-d))}{T^{-}(\Theta(-d))},
$$

where $d$ is defined in (31), then Assumption 3 is satisfied. 
The threshold depends on the distortions via $T^{+}$and $T^{-}$and on the asset returns via $d$ and $\Theta$. Note that it has appealing sensitivities with respect to exponential utility exponents: It is decreasing in $v$ and increasing in $\xi$. This is in line with intuition: Increasing $v$ causes a higher risk aversion on gains-only gambles. This gives high gains a lower value to the agent, which makes scaling up the position less attractive. Hence the optimal portfolio is more likely to be finite, which allows a lower threshold for Assumption 3 to be satisfied. On the other hand, increasing $\xi$ yields higher risk seeking on loss-only gambles. This makes large losses less harmful to the agent, which makes scaling up the position more attractive. In order to still guarantee a finite optimal portfolio, loss aversion must be greater.

\section{Numerical study}

In order to illustrate our results we derive the optimal portfolio for real-world returns. Hu and Kercheval [18] consider a portfolio of five major US stocks and their daily returns between 7 January 2002 and 8 April 2005. After controlling for serial correlation of stock volatility by a GARCH filter the portfolio is fitted by six generalized hyperbolic distributions, among which the $t$ distribution achieves the best fit. Thus we consider the multivariate normal distribution and the $t$ distribution, i.e.

$$
y \sim N_{5}(\mu, \Sigma) \quad \text { or } \quad y \sim t_{5}(\mu, \Sigma, v) .
$$

We take the correlation matrix of the five stocks derived by Hu and Kercheval [18]:

$$
\Sigma=\left(\begin{array}{lllll}
(1.144) 10^{-4} & (5.026) 10^{-5} & (4.687) 10^{-4} & (2.285) 10^{-5} & (7.010) 10^{-5} \\
(5.026) 10^{-5} & (1.638) 10^{-4} & (5.973) 10^{-5} & (2.849) 10^{-5} & (6.050) 10^{-5} \\
(4.687) 10^{-5} & (5.973) 10^{-5} & (1.69) 10^{-4} & (3.158) 10^{-5} & (6.023) 10^{-5} \\
(2.285) 10^{-5} & (2.849) 10^{-5} & (3.158) 10^{-5} & (1.276) 10^{-4} & (2.961) 10^{-5} \\
(7.010) 10^{-5} & (6.050) 10^{-5} & (6.023) 10^{-5} & (2.961) 10^{-5} & (2.433) 10^{-4}
\end{array}\right)
$$

Whereas Hu and Kercheval [18] consider annualized daily log returns, we require annualized daily arithmetic excess returns. Fortunately, the difference between log and arithmetic returns is negligible for a one day horizon. As reference point we consider the riskless return $r$. Note that the US dollar LIBOR has an average of $3.262 \%$ for the year 2005 and an average of $2.824 \%$ for April 2005. Thus we set the riskless daily return for 8 April 2005 to $r=3 \%$ and have the mean vector of excess returns $y=\bar{x}$

$$
\mu=[0.010,0.043,-0.045,0.009,-0.017] .
$$

Finally Hu and Kercheval [18] estimate $v=5.87$. Having specified these empirical returns, we have the optimal holding in the mean-variance-portfolio, given by

$$
\zeta_{M}=[102.54,390.1,-433.05,81.91,-41.64] \text {. }
$$

The highest amount is invested into the second stock Exxon, which has the highest expected return, whereas the third stock Pfizer has a short position, as its expected return is negative.

Concerning the CPT-specification, we set loss aversion $\lambda=2.25$, as estimated by Tversky and Kahneman [33]. For distortions we consider the form suggested by Prelec [30] in (3) and set $\gamma=0.63$, as estimated by Wu and Gonzalez [34], and $\delta_{1}=2$ and $\delta_{2}=1.5$. Proposition 1 thus yields Assumption 1 for our normal and $t$-distributed returns. Let us now consider two families of CPT-agents, one with power utilities and one with exponential utilities, and 

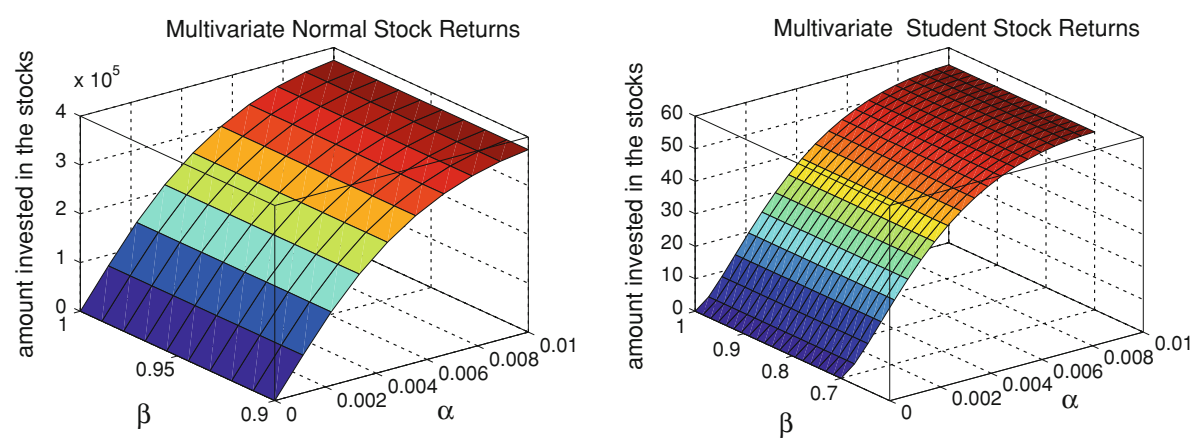

Fig. 1 The total amount $R$, a piecewise power CPT-agent invests into the stocks, plotted as a function of power utility exponents $\alpha$ and $\beta$. In the left panel we consider normally distributed stocks, whereas in the right panel stocks are $t$ distributed

derive the individual optimal participation in the mean-variance-portfolio. By Theorem 4 the total amount invested in the five risky stocks is

$$
R:=\sum_{i=0}^{n} \zeta_{i}^{*}=\sum_{i=0}^{n} k^{*} \zeta_{M, i} .
$$

For the piecewise power family of CPT-agents we leave the power exponents $\alpha$ and $\beta$ as free parameters. We plot the total amount invested in the stocks $R$ as a function of $\alpha$ and $\beta$ in Fig. 1.

Generally we find that the total amount invested in the stocks is very high for $\alpha>0.25$, e.g. the amount is of order $10^{7}$ for $\alpha=0.6$. One possible way to explain this high magnitude of $R$ is to observe that the mean-variance-portfolio is very attractive for the investor. It has a deviation in excess of the riskless return with a high expected value of $E D\left(\zeta_{M}\right)=\sigma_{M}^{2}=38.73$, while being exposed to low risk, as its standard deviation is $\sigma_{M}=6.2235$. Bernard and Ghossoub [6] also find high values of $R$ analytically for standard choices of $\alpha, \beta$ in a more extensive study with skew-normal returns. They further bound $R$ by the initial endowment $W_{0}$. Opposed to this we allow for borrowing, as this is possible to some extent in practice, and as it is delicate to specify the bound $W_{0}$. These high values of $R$ could be interpreted as a justification for regulating piecewise power agents by a risk constraint as in Section 3.2.

Our second observation is that $R$ is increasing in $\alpha$ and decreasing in $\beta$, which is consistent with intuition. We observe further that $R$ is highly depending on $\alpha$ and nearly insensitive to $\beta$. This can be explained as the gains depend on $\alpha$ and the losses depend on $\beta$. Indeed the prospect value in (4) is dominated by gains, whereas losses play a minor role. An intuitive argument for this could be the following: By the high mean $\sigma_{M}^{2}$, the cdf $F$ of the deviation of the mean-variance-portfolio puts most weight on the positive axis of gains, and little weight on the loss axis.

Third we observe, by comparing the two distributions, much higher values of $R$ for the normal returns compared to the $t$ returns. The reason for this might be that piecewise power agents are sensitive to the fatter tails of the $t$ distribution.

Finally we consider the second family of CPT-agents, who have a piecewise exponential value function. We plot total amount invested in the stocks $R$ as a function of exponential utility exponents $v$ and $\xi$ in Fig. 2. It contains only one plot for the normal distribution, as plots of the $t$ distribution look similar. The bounded value function seems to make piecewise exponential agent very insensitive to the tail behavior of the distribution. 


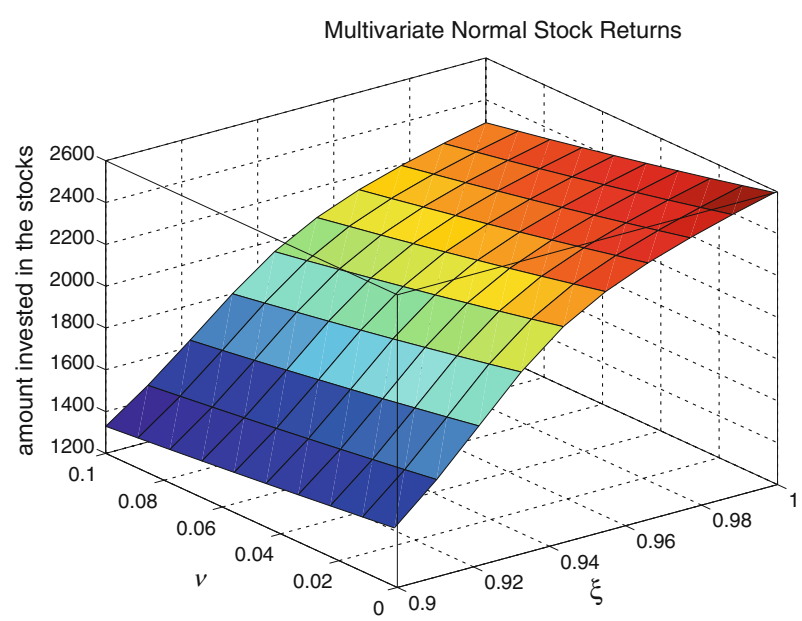

Fig. 2 The total amount $R$, a piecewise exponential CPT-agent invests into the normally-distributed stocks, plotted as a function of exponential utility exponents $v$ and $\xi$

Overall we find that the total optimal amounts invested in the stocks do not reach the excessive values of the piecewise power agents. An explanation for this might be that the piecewise exponential value function better mimics very high aversion to large-scale lotteries, which makes excessive investment unattractive.

Last, we again observe appealing sensitivities with respect to curvature parameters $v$ and $\xi$, since $R$ is decreasing in $v$ and increasing in $\xi$. This is in line with intuition given after Proposition 7.

\section{Appendix}

\subsection{Preparations}

Before proofing our results more generally, let us first consider the important special case of

$$
\zeta^{*}=\arg \max _{\zeta \in \mathbb{R}^{n}} V(D(\zeta))=\mathbf{0}
$$

The zero-portfolio is optimal, thus it is optimal not to deviate from the benchmark portfolio $a$. We have

$$
V(D(\zeta)) \leq V(D(\mathbf{0})) \text { for all } \zeta \neq \mathbf{0}
$$

where the prospect value of zero investment is just a number by

$$
V(D(\mathbf{0}))=V(-c)= \begin{cases}-\lambda u^{-}(c) & \text { for } c>0 \\ u^{+}(-c) & \text { for } c \leq 0 .\end{cases}
$$

In this case Theorem 4 holds trivially true with $k^{*}=0$ and Theorem 5 hold true with $\tilde{k}=0$. Propositions 1 and 2 are not effected by the form of $\zeta^{*}$ and proved below. Propositions 3, 5, and 7 hold true directly, as $\zeta^{*}=\mathbf{0}$ implies Assumption 3 trivially. In Proposition 4 we allow for $\zeta^{*}=\mathbf{0}$, but its proof below shows that $k=0$ is not optimal, as it leads to $V(0)=0$. For 
Proposition 6 we also allow for $\zeta^{*}=\mathbf{0}$ and it turns out to be optimal, as shown below. In economics it is common to exclude this case by a participation constraint, e.g. by assuming the existence of some $\zeta \in \mathbb{R}^{n}$ with

$$
V(D(\zeta))>V(D(0))
$$

For clarity let us state, that we do not exclude the case $\zeta^{*}=\mathbf{0}$, we just consider it separately for the ease of presentation.

Having completed the trivial case $\zeta^{*}=\mathbf{0}$, we consider henceforth

$$
\zeta^{*} \neq \mathbf{0}
$$

Thus we only need to consider $\zeta \neq \mathbf{0}$, when solving for $\zeta^{*}$. It follows that the variance of the deviation $\bar{\sigma}^{2}$ is strictly positive (which we will use frequently without notice)

$$
\bar{\sigma}^{2}=\zeta^{T} \Sigma \zeta>0
$$

since the covariance matrix is positive definite. In further preparation for the following proofs we recall the distorted density functions $f^{+}$and $f^{-}$defined in (19). They are strictly positive as the distortion functions $T^{+}$and $T^{-}$are strictly increasing:

$$
f^{+}(x)=-T_{x}^{+}(1-\Theta(x))(-\theta(x))>0, \quad f^{-}(x)=T_{x}^{-}(\Theta(x)) \theta(x)>0 .
$$

By the chain rule the prospect value of (4) writes as

$$
\begin{aligned}
V(D(\zeta))= & \int_{0}^{\infty} u^{+}(x)\left(-T^{+}\left(1-\Theta\left(\frac{x-\bar{\mu}}{\bar{\sigma}}\right)\right)\right)_{x} \mathrm{~d} x \\
& -\lambda \int_{-\infty}^{0} u^{-}(-x)\left(T^{-}\left(\Theta\left(\frac{x-\bar{\mu}}{\bar{\sigma}}\right)\right)\right)_{x} \mathrm{~d} x \\
= & \frac{1}{\bar{\sigma}}\left[\int_{0}^{\infty} u^{+}(x) f^{+}\left(\frac{x-\bar{\mu}}{\bar{\sigma}}\right) \mathrm{d} x-\lambda \int_{-\infty}^{0} u^{-}(-x) f^{-}\left(\frac{x-\bar{\mu}}{\bar{\sigma}}\right) \mathrm{d} x .\right] \\
= & : v^{+}(\bar{\mu}, \bar{\sigma})-\lambda v^{-}(\bar{\mu}, \bar{\sigma})=: v(\bar{\mu}, \bar{\sigma}) .
\end{aligned}
$$

We see that the prospect value is a function of the portfolio's mean and variance. Further it is strictly increasing in the portfolio's mean return:

Proposition 8 The function $\bar{\mu} \rightarrow v(\bar{\mu}, \cdot)$ is strictly increasing.

Proof We first show that $\bar{\mu} \rightarrow v^{+}(\bar{\mu}, \cdot)$ is strictly increasing. A change of variable gives

$$
v^{+}(\bar{\mu}, \bar{\sigma})=\left[\int_{\frac{-\bar{\mu}}{\bar{\sigma}}}^{\infty} u^{+}(\bar{\sigma} y+\bar{\mu}) f^{+}(y) \mathrm{d} y\right] .
$$

Since $u^{+}(\bar{\sigma} y+\bar{\mu})$ is increasing in $\bar{\mu}, f^{+}$and $u^{+}$are strictly positive, and the integration limit $\frac{-\bar{\mu}}{\bar{\sigma}}$ is strictly decreasing in $\bar{\mu}$, the claim follows. By the same argument $v^{-}(\bar{\mu}, \bar{\sigma})$ is strictly decreasing in $\bar{\mu}$. 


\subsection{Proof of Proposition 1}

This proposition is proved by He and Zhou [17] in the case of the normal and lognormal distribution. It remains to show the claim the $t$ distribution. Following He and Zhou [17] it suffices to show that

$$
\begin{array}{r}
T_{x}^{+}(F(x)) f(x)=O\left(|x|^{-2-\epsilon}\right), \quad T_{x}^{-}(F(x)) f(x)=O\left(|x|^{-2-\epsilon}\right), \\
T_{x}^{+}(1-F(x)) f(x)=O\left(|x|^{-2-\epsilon}\right), \quad T_{x}^{+}(1-F(x)) f(x)=O\left(|x|^{-2-\epsilon}\right),
\end{array}
$$

for some $\epsilon>0$ and sufficiently large $|x|$ and $0<F(x)<1$. This claim follows in the light of property (5), since for the cdf of the $t$ distribution with $v$ degrees of freedom it holds

$$
F(x)=O\left(|x|^{\nu-1}\right), \quad 1-F(x)=O\left(|x|^{\nu-1}\right) .
$$

\subsection{Proof of Theorem 4}

By Assumption 3, $\zeta^{*} \in \mathbb{R}^{n}$ is a critical point. Thus it satisfies the following first-order condition

$$
\nabla_{\zeta} V\left(D\left(\zeta^{*}\right)\right)=\mathbf{0}
$$

Observe that the portfolio $\zeta$ enters the prospect value of (24) only via the mean $\bar{\mu}(\zeta)$ and the standard deviation $\bar{\sigma}(\zeta)$. Thus the total differential takes the simple form

$$
\nabla_{\zeta} V(D(\zeta))=\nabla_{\zeta} v(\bar{\mu}(\zeta), \bar{\sigma}(\zeta))=v_{\bar{\mu}} \nabla_{\zeta} \bar{\mu}(\zeta)+v_{\bar{\sigma}} \nabla_{\zeta} \bar{\sigma}(\zeta)
$$

Since the gradients of the mean and the variance are

$$
\nabla_{\zeta} \bar{\mu}(\zeta)=\mu, \quad \nabla_{\zeta} \bar{\sigma}(\zeta)=\nabla_{\zeta} \sqrt{\zeta^{T} \Sigma \zeta}=\frac{2 \Sigma \zeta}{2 \bar{\sigma}}=\bar{\sigma}^{-1} \Sigma \zeta
$$

the first-order condition writes as

$$
\mathbf{0}=\nabla_{\zeta} V(D(\zeta))=v_{\bar{\mu}} \mu+v_{\bar{\sigma}} \bar{\sigma}^{-1} \Sigma \zeta^{*} .
$$

Provided that $v_{\bar{\sigma}} \neq 0$, we can solve for the optimal portfolio

$$
\zeta^{*}=-\frac{\bar{\sigma} v_{\bar{\mu}}}{v_{\bar{\sigma}}} \Sigma^{-1} \mu=\kappa\left(\zeta^{*}\right) \zeta_{M}
$$

where $\kappa\left(\zeta^{*}\right)=-\frac{\bar{\sigma} v_{\bar{\mu}}\left(\bar{\mu}\left(\zeta^{*}\right), \bar{\sigma}\left(\zeta^{*}\right)\right)}{v_{\bar{\sigma}}\left(\bar{\mu}\left(\zeta^{*}\right), \bar{\sigma}\left(\zeta^{*}\right)\right)} \in \mathbb{R}$. It remains to show that $v_{\bar{\sigma}} \neq 0$. Assuming the contrary $v_{\bar{\sigma}}=0$, it follows

$$
v_{\bar{\mu}}=0
$$

by the first-order condition in (27) provided $\mu \neq \mathbf{0}$. We can assume $\mu \neq \mathbf{0}$ without loss of generality, else the maximization problem is trivially solved by $\zeta^{*}=\mathbf{0}$, in which case Eq. (28) holds independent of $v_{\bar{\sigma}} \neq 0$. However, by Proposition 8 it holds that

$$
v_{\bar{\mu}}>0
$$

which contradicts Eq. (29) and thus establishes the remaining inequality $v_{\bar{\sigma}} \neq 0$. In consequence we have shown that the optimal portfolio is of the form $k^{*} \zeta_{M}$, where $k^{*}$, as defined in (12), is finite by Assumption 3. 


\subsection{Proof of Theorem 5}

The existence of $\zeta_{K}^{*}$, which is defined in (17), follows as we optimize the differentiable function $V(D(\zeta))$ over the compact set $K$. There are two cases for $\zeta_{K}^{*}$ : Either it is not a regular point, which means that

$$
\nabla_{\zeta} \rho(\bar{\mu}, \bar{\sigma})=\mathbf{0}
$$

or else there exists a nonnegative Lagrange multiplier $\lambda$ such that

$$
\nabla_{\zeta} v(\bar{\mu}, \bar{\sigma})-\lambda \nabla_{\zeta} \rho(\bar{\mu}, \bar{\sigma})=\mathbf{0}
$$

In the first case the total differential of the constraint writes by (26) as

$$
\nabla_{\zeta} \rho(\bar{\mu}, \bar{\sigma})=\rho_{\bar{\mu}} \mu+\rho_{\bar{\sigma}} \bar{\sigma}^{-1} \Sigma \zeta_{K}^{*}=\mathbf{0},
$$

which by $\rho_{\bar{\sigma}} \neq 0$ provides the claim by

$$
\zeta_{K}^{*}=\eta \Sigma^{-1} \mu, \quad \eta=-\frac{\bar{\sigma} \rho_{\bar{\mu}}}{\rho_{\bar{\sigma}}} .
$$

In the second case the total differential is

$$
\left[v_{\bar{\mu}}-\lambda \rho_{\bar{\mu}}\right] \mu+\bar{\sigma}^{-1}\left[v_{\bar{\sigma}}-\lambda \rho_{\bar{\sigma}}\right] \Sigma \zeta_{K}^{*}=\mathbf{0} .
$$

Provided $v_{\bar{\sigma}}-\lambda \rho_{\bar{\sigma}} \neq 0$ the claim follows by

$$
\zeta_{K}^{*}=\eta \Sigma^{-1} \mu, \quad \eta=-\frac{\bar{\sigma} v_{\bar{\mu}}-\lambda \bar{\sigma} \rho_{\bar{\mu}}}{v_{\bar{\sigma}}-\lambda \rho_{\bar{\sigma}}} .
$$

It remains to show

$$
v_{\bar{\sigma}}-\lambda \rho_{\bar{\sigma}} \neq 0
$$

Assuming the contrary $v_{\bar{\sigma}}-\lambda \rho_{\bar{\sigma}}=0$, it follows by the first-order condition, $\bar{\sigma}>0$, and $\mu \neq \mathbf{0}$ (without loss of generality)

$$
v_{\bar{\mu}}-\lambda \rho_{\bar{\mu}}=0,
$$

which is a contradiction to $\lambda \geq 0, \rho_{\bar{\mu}}<0$, and $v_{\bar{\mu}}>0$ from Proposition 8 . In both cases the optimal portfolio is a scalar of the mean-variance-portfolio $\zeta_{M}$. Thus it is given by

$$
\zeta_{K}^{*}=\tilde{k} \zeta_{M}
$$

with $\tilde{k}$ defined in (16).

\subsection{Proof of Proposition 2}

This proposition is proved in Barberis and Huang [3], who assume $\alpha=\beta$ and $\delta=\gamma$. Thus the required condition writes as $\alpha<2 \delta$, which is already noted by Bernard and Ghossoub [6]. 


\subsection{Proof of Proposition 3}

We substitute $z=x / \bar{\sigma}$ in (24) and factor out the loss integral to get

$$
\begin{aligned}
V(D(\zeta)) & =\int_{0}^{\infty} \bar{\sigma}^{\alpha} z^{\alpha} f^{+}\left(z-\frac{\bar{\mu}}{\bar{\sigma}}\right) \mathrm{d} z-\lambda \int_{-\infty}^{0} \bar{\sigma}^{\beta}(-z)^{\beta} f^{-}\left(z-\frac{\bar{\mu}}{\bar{\sigma}}\right) \mathrm{d} z \\
& =\bar{\sigma}^{\beta} \lambda L(\bar{\mu}, \bar{\sigma})\left[\bar{\sigma}^{\alpha-\beta} \frac{G(\bar{\mu}, \bar{\sigma})}{\lambda L(\bar{\mu}, \bar{\sigma})}-1\right],
\end{aligned}
$$

where $G(\bar{\mu}, \bar{\sigma}):=\int_{0}^{\infty} z^{\alpha} f^{+}\left(z-\frac{\bar{\mu}}{\bar{\sigma}}\right) \mathrm{d} z, L(\bar{\mu}, \bar{\sigma}):=\int_{-\infty}^{0}(-z)^{\beta} f^{-}\left(z-\frac{\bar{\mu}}{\bar{\sigma}}\right) \mathrm{d} z$.

It remains to show that gains $G(\bar{\mu}, \bar{\sigma})$ have an upper bound independent of $\zeta$, and that losses $L(\bar{\mu}, \bar{\sigma})$ have a lower positive bound independent of $\zeta$ if $\bar{\sigma}$ is large enough. Then (18) follows by (30) and the assumption $\alpha<\beta$, since the square bracket is strictly negative and $\bar{\sigma}$ is strictly positive. Finally, Assumption 3 follows by (18) and $V(D(\mathbf{0}))=V(-c)$.

To see that $G(\bar{\mu}, \bar{\sigma})$ is bounded independent of $\zeta$, we note that it holds $\bar{\sigma}^{2}=\zeta^{T} \Sigma \zeta \geq$ $q|\zeta|^{2}$ for some positive constant $q$ as the covariance matrix is positive semi-definite. Thus it follows by the Cauchy-Schwarz inequality that the ratio

$$
\frac{|\bar{\mu}(\zeta)|}{\bar{\sigma}(\zeta)} \leq \frac{|\mu||\zeta|}{\sqrt{q}|\zeta|}=\frac{|\mu|}{\sqrt{q}}=: d,
$$

is bounded independent of $\zeta$. By substituting $y=z-\frac{\bar{\mu}}{\bar{\sigma}}$ and the positive derivative with respect to $\frac{\bar{\mu}}{\bar{\sigma}}$ we find by $\frac{\bar{\mu}}{\bar{\sigma}} \leq d$

$$
G(\bar{\mu}, \bar{\sigma})=\int_{-\frac{\bar{\mu}}{\bar{\sigma}}}^{\infty}\left(y+\frac{\bar{\mu}}{\bar{\sigma}}\right)^{\alpha} f^{+}(y) \mathrm{d} y \leq \int_{-d}^{\infty}(y+d)^{\alpha} f^{+}(y) \mathrm{d} y .
$$

The latter integral can be expressed by the prospect value of gains $V^{+}$of a portfolio $\zeta_{d}$, for which the deviation has mean $\bar{\mu}\left(\zeta_{d}\right)=d$ and standard deviation $\bar{\sigma}\left(\zeta_{d}\right)=1$, which gives us

$$
G(\bar{\mu}, \bar{\sigma}) \leq \int_{0}^{\infty} z^{\alpha} f^{+}(z-d) \mathrm{d} y=\frac{1}{\bar{\sigma}\left(\zeta_{d}\right)} V^{+}\left(D\left(\zeta_{d}\right)\right)<\infty .
$$

Finally note that $V^{+}\left(D\left(\zeta_{d}\right)\right)$ is bounded independent of $\zeta$ by Assumption 1. An analogous argument shows that

$$
L(\bar{\mu}, \bar{\sigma}) \geq V^{-}\left(D\left(\zeta_{d}\right)\right)=\int_{-\infty}^{0}(-z)^{\beta} f^{-}(z-d) \mathrm{d} y,
$$

which is strictly positive by the positivity of $f^{-}$.

\subsection{Proof of Proposition 4}

Under $\alpha<\beta$ it follows Assumption 3 by Proposition 3. Assumption 3 implies the two-fund separation and it only remains to solve for $k^{*}$. Notice that the prospect value of the mean-variance-portfolio $V\left(D\left(k \zeta_{M}\right)\right)$ is a one-dimensional function in $k$. We consider its two branches 
on the positive and the negative axis separately. Further we use the following moments

$$
\bar{\mu}\left(k \zeta_{M}\right)=k E D\left(\zeta_{M}\right)=k\left(\sigma_{M}^{2}-c\right)=k \sigma_{M}^{2}, \quad \bar{\sigma}^{2}\left(k \zeta_{M}\right)=\operatorname{Var}\left(k D\left(\zeta_{M}\right)\right)=k^{2} \sigma_{M}^{2} .
$$

For $k>0$, we substitute $y=\frac{x}{k}$ in (24) to obtain

$$
\begin{aligned}
V\left(D\left(k \zeta_{M}\right)\right)= & \frac{1}{\sigma_{M}} k^{\alpha} \int_{0}^{\infty} y^{\alpha} f^{+}\left(\frac{y}{\sigma_{M}}-\sigma_{M}\right) \mathrm{d} y \\
& -\frac{1}{\sigma_{M}} \lambda k^{\beta} \int_{-\infty}^{0}(-y)^{\beta} f^{-}\left(\frac{y}{\sigma_{M}}-\sigma_{M}\right) \mathrm{d} y
\end{aligned}
$$

Note that the above two integrals are independent of $k$, so the positive branch is polynomial in $k$. Thus it has a unique critical point: the positive $k_{1}$ as defined in (21). On the negative branch with $k<0$, we substitute $y=-\frac{x}{k}$ in (24) to obtain

$$
\begin{aligned}
V\left(D\left(k \zeta_{M}\right)\right)= & \frac{-k}{|k| \sigma_{M}}(-k)^{\alpha} \int_{0}^{\infty} y^{\alpha} f^{+}\left(-\frac{y}{\sigma_{M}}-\sigma_{M}\right) \mathrm{d} y \\
& +\frac{1}{\sigma_{M}} \lambda(-k)^{\beta} \int_{-\infty}^{0}(-y)^{\beta} f^{-}\left(-\frac{y}{\sigma_{M}}-\sigma_{M}\right) \mathrm{d} y
\end{aligned}
$$

The negative polynomial branch has the unique critical point $k_{2}$ defined in (22). By $\alpha<\beta$ and the proof of (18) it holds

$$
\lim _{|k| \rightarrow \infty} V\left(D\left(k \zeta_{M}\right)\right)=-\infty
$$

Consequently the prospect value $V\left(D\left(k \zeta_{M}\right)\right)$ attains its maximum at one of the two suggested finite critical points, which closes the proof.

\subsection{Proof of Proposition 5}

The prospect value of an arbitrary portfolio (30) writes as

$$
V(D(\zeta))=\bar{\sigma} \lambda L(\bar{\mu}, \bar{\sigma})\left[\frac{G(\bar{\mu}, \bar{\sigma})}{\lambda L(\bar{\mu}, \bar{\sigma})}-1\right]
$$

in the case of linear utilities, where

$$
G(\bar{\mu}, \bar{\sigma}):=\int_{0}^{\infty} z f^{+}\left(z-\frac{\bar{\mu}}{\bar{\sigma}}\right) \mathrm{d} z, \quad L(\bar{\mu}, \bar{\sigma}):=\int_{-\infty}^{0}(-z) f^{-}\left(z-\frac{\bar{\mu}}{\bar{\sigma}}\right) \mathrm{d} z .
$$

As in (32) and (33) we have $G(\bar{\mu}, \bar{\sigma}) \leq V^{+}\left(D\left(\zeta_{d}\right)\right)$ and $L(\bar{\mu}, \bar{\sigma}) \geq V^{-}\left(D\left(\zeta_{d}\right)\right)$. Thus the assumption on $\lambda$ implies

$$
\frac{G(\bar{\mu}, \bar{\sigma})}{\lambda L(\bar{\mu}, \bar{\sigma})}-1<0 .
$$

This condition in (34) yields (18), which in turn ensures Assumption 3. 


\subsection{Proof of Proposition 6}

Assumption 3 implies the two-fund separation by Theorem 4 and the existence of a finite $k^{*}$ with

$$
V\left(D\left(k^{*} \zeta_{M}\right)\right)=\max _{k \in \mathbb{R}} V\left(D\left(k \zeta_{M}\right)\right)=\max _{\zeta \in \mathbb{R}^{n}} V(D(\zeta))
$$

For $k>0$ we find again by substituting $y=\frac{x}{k}$

$$
V\left(D\left(k \zeta_{M}\right)\right)=\frac{k}{\sigma_{M}}\left[\int_{0}^{\infty} y f^{+}\left(\frac{y}{\sigma_{M}}-\sigma_{M}\right) \mathrm{d} y-\lambda \int_{-\infty}^{0}(-y) f^{-}\left(\frac{y}{\sigma_{M}}-\sigma_{M}\right) \mathrm{d} y\right],
$$

which is linear in $k$. The square bracket, which is independent of $k$, cannot be positive in the light of a finite $k^{*}$ with (35), which implies the first stated condition on $\lambda$ for $k=1$. Analogously, for $k<0$ the substitution $y=\frac{-x}{k}$ implies the other condition on $\lambda$ for $k=-1$. Overall, we see that for $k \neq 0$ the prospect value $V\left(D\left(k \zeta_{M}\right)\right)$ cannot be positive, and is thus maximized by $k^{*}=0$, which gives a prospect value of $V(D(\mathbf{0}))=0$.

\subsection{Proof of Proposition 7}

We substitute $z=x / \bar{\sigma}$ in (24) and insert the exponential utilities

$$
V(D(\zeta))=\int_{0}^{\infty} \frac{1}{\nu}\left(1-e^{-\nu \bar{\sigma} x}\right) f^{+}\left(z-\frac{\bar{\mu}}{\bar{\sigma}}\right) \mathrm{d} z-\lambda \int_{-\infty}^{0} \frac{1}{\xi}\left(1-e^{\xi \bar{\sigma} x}\right) f^{-}\left(z-\frac{\bar{\mu}}{\bar{\sigma}}\right) \mathrm{d} z .
$$

For all $\epsilon>0$ there exists $\bar{\sigma}$ large enough such that

$$
\begin{aligned}
V(D(\zeta)) & <\int_{0}^{\infty} \frac{1}{v} f^{+}\left(z-\frac{\bar{\mu}}{\bar{\sigma}}\right) \mathrm{d} z-\lambda \int_{-\infty}^{0} \frac{1}{\xi}(1-\epsilon) f^{-}\left(z-\frac{\bar{\mu}}{\bar{\sigma}}\right) \mathrm{d} z \\
& =\int_{-\frac{\bar{\mu}}{\bar{\sigma}}}^{\infty} \frac{1}{v} f^{+}(x) \mathrm{d} x-\lambda \int_{-\infty}^{-\frac{\bar{\mu}}{\bar{\sigma}}} \frac{1}{\xi}(1-\epsilon) f^{-}(x) \mathrm{d} x
\end{aligned}
$$

By the equalities $\int_{a}^{b} f^{+}(x) \mathrm{d} x=-T^{+}(1-\Phi(b))+T^{+}(1-\Phi(a))$ and $\int_{a}^{b} f^{-}(x) \mathrm{d} x=$ $T^{-}(\Phi(b))-T^{-}(\Phi(a))$ we get

$$
V(D(\zeta))<\frac{1}{\nu}\left[T^{+}\left(1-\Theta\left(-\frac{\bar{\mu}}{\bar{\sigma}}\right)\right)-T^{+}(0)\right]-\lambda \frac{1}{\xi}(1-\epsilon)\left[T^{-}\left(\Theta\left(-\frac{\bar{\mu}}{\bar{\sigma}}\right)\right)-T^{-}(0)\right] .
$$

Further the equality $\frac{\bar{\mu}}{\bar{\sigma}} \leq d$ for all $\zeta$ by (31), the monotonicity of the functions $\Theta, T^{+}$, and $T^{-}$, and $T^{+}(0)=0=T^{-}(0)$ imply

$$
V(D(\zeta))<\frac{1}{v} T^{+}(1-\Theta(-d))-\lambda \frac{1}{\xi}(1-\epsilon) T^{-}(\Theta(-d)) .
$$

We observe that the right hand side of the inequality is independent of $\bar{\sigma}$ and let $\epsilon$ approach 0 to get

$$
\lim \sup _{|\zeta| \rightarrow \infty} V(D(\zeta)) \leq \frac{1}{v} T^{+}(1-\Theta(-d))-\lambda \frac{1}{\xi} T^{-}(\Theta(-d))
$$




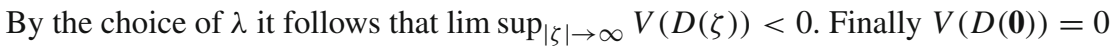

Acknowledgments This work is supported by NSERC Grant 371653-09, MITACS Grant 5-26761, SSHRC Grant 5-26758, and the National Centre of Competence in Research "Financial Valuation and Risk Management" (NCCR FINRISK). The authors would like to thank two anonymous referees for numerous helpful comments and Huayue Zhang for her help with the numerical illustration.

\section{References}

1. Allais, M.: Le comportement de l'homme rationnel devant le risque: critique des postulats et axiomes de l'école Américaine. Econometrica 21(4), 503-546 (1953)

2. Aparicio, F.M., Estrada, J.: Empirical distributions of stock returns: European securities markets. Eur. J. Financ 7(1), 1-21 (2001)

3. Barberis, N., Huang, M.: Stocks as lotteries: the implications of probability weighting for security prices. Am. Econ. Rev. 98(5), 2066-2100 (2008)

4. Barndorff-Nielsen, O., Kent, J., Sørensen, M.: Normal variance-mean mixtures and $z$ distributions. Int. Statist. Rev. 50(2), 145-159 (1982)

5. Benartzi, S., Thaler, R.H.: Myopic loss aversion and the equity premium puzzle. Quart. J. Econ. 110(1), 73-92 (1995)

6. Bernard, C., Ghossoub, M.: Static portfolio choice under cumulative prospect theory. Math. Financ Econ. 2, 77-306 (2010)

7. Bingham, N.H., Kiesel, R.: Semi-parametric modelling in finance: theoretical foundations. Quant. Financ 2(4), 241-250 (2002)

8. Bingham, N.H., Kiesel, R., Schmidt, R.: A semi-parametric approach to risk management. Quant. Financ. 3(6), 426-441 (2003)

9. Blattberg, R.C., Gonedes, N.J.: A comparison of the stable and student distributions as statistical models for stock prices. J. Bus. 47(2), 244-280 (1974)

10. Blondel, S.: Testing theories of choice under risk: estimation of individual functionals. J. Risk Uncertain. 24(3), 251-265 (2002)

11. Chamberlain, G.: A characterization of the distributions that imply mean-variance utility functions. J. Econ. Theory 29, 185-201 (1983)

12. De Giorgi, E., Hens, T., Levy, H.: Existence of CAPM equilibria with prospect theory preferences. Working paper, University of St. Gallen, St. Gallen (2011)

13. De Giorgi, E., Hens, T., Mayer, J.: A note on reward-risk portfolio and two-fund separation. Financ. Res. Lett. 29, 185-201 (2011)

14. Eberlein, E., Keller, U.: Hyperbolic distributions in finance. Bernoulli 1(3), 281-299 (1995)

15. Ellsberg, D.: Risk, ambiguity, and the Savage axioms. Quart. J. Econ. 75(4), 643-669 (1961)

16. Fang, K.T., Kotz, S., Ng, K.W.: Symmetric multivariate and related distributions. Chapman and Hall, London (1990)

17. He, X.D., Zhou, X.: Portfolio choice under cumulative prospect theory: an analytical treatment. Manag. Sci. 57(2), 315-331 (2011)

18. Hu, W., Kercheval, A.: Portfolio optimization for student $t$ and skewed $t$ return. Quant. Financ. 10(1), 91-105 (2010)

19. Hull, j., White, A.: Value at risk when daily changes in market variables are not normally distributed. J. Deriv. 5, 9-19 (1998)

20. Hurst, S.R., Platen, E.: The marginal distribution of returns and volatility. IMS Lect. Notes Monogr. Ser. 31, 301-314 (1997)

21. Ingersoll, J.: Non-monotonicity of the Tversky-Kahneman probability-weighting function: a cautionary note. Eur. Financ. Manag. 14(3), 385-390 (2008)

22. Kahneman, D., Tversky, A.: Prospect theory: an analysis of decisions under risk. Econometrica 47, 263-291 (1979)

23. Köbberling, V., Wakker, P.: An index of loss aversion. J. Econ. Theory 122, 119-131 (2005)

24. Kusuoka, S.: On law-invariant coherent risk measures. Adv. Math. Econ. 3, 83-95 (2001)

25. Levy, H., Duchin, R.: Asset return distributions and the investment horizon. J. Portf. Manag. 30(3), 47-62 (2004)

26. Madan, D.B., Seneta, E.: The variance gamma (VG) model for share market returns. J. Bus. 63(4), 511-524 (1990)

27. Markowitz, H.: Portfolio selection. J. Financ. 7, 77-91 (1952) 
28. Owen, J., Rabinovitch, R.: On the class of elliptical distributions and their applications to the theory of portfolio choice. J. Financ. 38, 745-752 (1983)

29. Praetz, P.D.: The distribution of share price changes. J. Bus. 45(1), 49-55 (1972)

30. Prelec, D.: Portfolio optimization for $t$ and skewed $t$ returns. Quant. Financ. 10, 91-105 (1998)

31. Rieger, M.O., Bui, T.: Too risk-averse for prospect theory?. Mod. Econ. 2(4), 691-700 (2011)

32. Tobin, J.: Liquidity preference as behavior towards risk. Rev. Econ. Stud. 25, 65-86 (1958)

33. Tversky, A., Kahneman, D.: Advances in prospect theory: cumulative representation of uncertainty. J. Risk Uncertain. 5(4), 297-323 (1992)

34. Wu, G., Gonzalez, R.: Curvature of the probability weighting function. Manag. Sci. 42(12), 16761690 (1996) 